\title{
Corrigendum: Innate Immune Cells in the Esophageal Tumor Microenvironment
}

\begin{abstract}
Kele Cui ${ }^{1,3,4 * t}$, Shouxin $\mathrm{Hu}^{2,3,4 t}$, Xinyu Mei ${ }^{5}$ and Min Cheng ${ }^{2,3,4 *}$
${ }^{1}$ Department of Clinical Laboratory, The First Affiliated Hospital of USTC, Division of Life Sciences and Medicine, University of Science and Technology of China, Hefei, China, ${ }^{2}$ Department of Geriatrics, Gerontology Institute of Anhui Province, The First Affiliated Hospital of USTC, Division of Life Sciences and Medicine, University of Science and Technology of China, Hefei, China, ${ }^{3}$ Anhui Provincial Key Laboratory of Tumor Immunotherapy and Nutrition Therapy, Hefei, China, ${ }^{4}$ Cancer Immunotherapy Center, The First Affiliated Hospital of USTC, Division of Life Sciences and Medicine, University of Science and Technology of China, Hefei, China, ${ }^{5}$ Department of Thoracic Surgery, The First Affiliated Hospital of USTC, Division of Life Sciences and Medicine, University of Science and Technology of China, Hefei, China
\end{abstract}

Keywords: innate immune cells, crosstalk, regulation, esophageal tumor microenvironment, immunotherapy strategy

\section{A Corrigendum on}

Innate Immune Cells in the Esophageal Tumor Microenvironment by Cui K, Hu S, Mei X and Cheng M (2021). Front. Immunol. 12:654731. doi: 10.3389/ fimmu.2021.654731

Approved by:

Frontiers Editorial Office, Frontiers Media SA, Switzerland

*Correspondence: Kele Cui ckele@mail.ustc.edu.cn Min Cheng

chengmin@ustc.edu.cn

${ }^{\dagger}$ These authors have contributed equally to this work

Specialty section:

This article was submitted to Molecular Innate Immunity,

a section of the journal

Frontiers in Immunology

Received: 12 May 2021 Accepted: 14 May 2021

Published: 27 May 2021

Citation:

Cui K, Hu S, Mei $X$ and Cheng M (2021) Corrigendum: Innate Immune Cells in the Esophageal Tumor Microenvironment.

Front. Immunol. 12:708705. doi: 10.3389/fimmu.2021.708705 Clinical Laboratory, Division of Life Sciences and Medicine, The First Affiliated Hospital of USTC, University of Science and Technology of China, Hefei, China; ${ }^{2}$ Department of Geriatrics, Gerontology Institute of Anhui Province, Division of Life Sciences and Medicine, The First Affiliated Hospital of USTC, University of Science and Technology of China, Hefei, China; ${ }^{4}$ Cancer Immunotherapy Center, Division of Life Sciences and Medicine, The First Affiliated Hospital of USTC, University of Science and Technology of China, Hefei, China; ${ }^{5}$ Department of Thoracic Surgery, Division of Life Sciences and Medicine, The First Affiliated Hospital of USTC, University of Science and Technology of China, Hefei, China", it should be "1 Department of Clinical Laboratory, the First Affiliated Hospital of USTC, Division of Life Sciences and Medicine, University of Science and Technology of China, Hefei, China; ${ }^{2}$ Department of Geriatrics, Gerontology Institute of Anhui Province, the First Affiliated Hospital of USTC, Division of Life Sciences and Medicine, University of Science and Technology of China, Hefei, China; ${ }^{4}$ Cancer Immunotherapy Center, the First Affiliated Hospital of USTC, Division of Life Sciences and Medicine, University of Science and Technology of China, Hefei, China; ${ }^{5}$ Department of Thoracic Surgery, the First Affiliated Hospital of USTC, Division of Life Sciences and Medicine, University of Science and Technology of China, Hefei, China".

The authors apologize for this error and state that this does not change the scientific conclusions of the article in any way. The original article has been updated.

Copyright $(02021 \mathrm{Cui}, \mathrm{Hu}, \mathrm{Mei}$ and Cheng. This is an open-access article distributed under the terms of the Creative Commons Attribution License (CC BY). The use, distribution or reproduction in other forums is permitted, provided the original author(s) and the copyright owner(s) are credited and that the original publication in this journal is cited, in accordance with accepted academic practice. No use, distribution or reproduction is permitted which does not comply with these terms.
In the published article, there was an error in affiliations 1, 2, 4 and 5. Instead of "1 Department of 